CCASE REPORT

Volume 16 Supp 12021

DOI: 10.21315/aos2021.16.s1.12

ARTICLE INFO

Submitted: 02/03/2021

Accepted: 27/05/2021

Online: 22/09/2021

\section{Challenges in Diagnosis and Therapy of Recurrent Oral Herpes Infection: Study of Two Cases}

\author{
Dwi Kartika Sari*, Febrina Rahmayanti, Harum Sasanti, \\ Ambar Kusuma Astuti \\ Department of Oral Medicine, Faculty of Dentistry, \\ Universitas Indonesia, Fakarta 10430, Indonesia \\ *Corresponding author: dwikartika113@gmail.com
}

To cite this article: Sari DK, Rahmayanti F, Sasanti H, Astuti AK (2021). Challenges in diagnosis and therapy of recurrent oral herpes infection: Study of two cases. Arch Orofac Sci, 16(Supp. 1): 73-81. https://doi.org/10.21315/aos2021.16.s1.12

To link to this article: https://doi.org/10.21315/aos2021.16.s1.12

\begin{abstract}
The symptoms of recurrent oral herpes infection may vary, from mild discomfort to life threatening. Dentists are more likely to be consulted in this oral infection, hence the ability to diagnose and treat this disease is mandatory. This article described manifestation and therapy of recurrent oral herpes infection. In the first case, a 41-year-old woman came with a complaint of painful sore mouth in her lower gingiva. She experienced canker sores for two to three times a year on the tongue, lips and palate. Laboratory results showed positive IgM anti HSV-2, which matched with the primary herpes infection. However, based on the history and clinical manifestations, she was diagnosed with recurrent intra oral herpes infection. In the second case, a 70-year-old man came with a very painful canker sores in his entire mouth. The patient had to be hospitalised for three days and received analgesic, antibiotic and gel containing triamcinolone. After hospitalisation, canker sores did not heal. Extraorally, we found a crust in the vermillion border of the upper lip and intraorally, we found multiple ulceration of keratinised and non-keratinised mucosa. The use of topical steroid in this patient may aggravated ulceration due to its ability to cause rapid spreading of the virus. The first patient was given chlorhexidine gluconate $0.2 \%$ and the second patient was prescribed with doxycycline rinse. Both patients received multivitamin containing zinc. Complete history taking, objective and adjunctive examination played a role in establishing the diagnosis and treatment of recurrent oral herpes infections.
\end{abstract}

Keywords: Oral manifestation; recurrent intra oral herpes infection; therapy

\section{INTRODUCTION}

Orofacial diseases in human are mainly caused by the herpes simplex virus (HSV). There are two immunologically different types of herpes simplex virus, namely HSV type 1 (HSV-1) which usually occurs in the lips, face, oral cavity and upper body area; and the second one, HSV type 2 (HSV-2) which usually occurs in the genital and lower body areas (Shah et al., 2014). Oral herpes simplex infection can manifest as primary or secondary (recurrent) infection (van der Plas \& Hardie, 2010). In the oral cavity, herpes virus infection occurs in three clinical types. First, the presence of recurrent small blisters on the lips known as recurrent herpes labialis. Second, a general oral infection known as primary herpetic stomatitis, and the third is recurrent intra oral herpes consisting of small vesicles and/or ulcer that usually occurs in the nonkeratinised area (Bhateja et al., 2017). In general, the primary infection will be more severe accompanied by enlarged 
lymph nodes, fever and discomfort (Chauvin \& Ajar, 2002). The dentist must be able to recognise and differentiate the primary or recurrent forms of oral herpes infections and distinguish them from other diseases that have similar clinical appearance. In this article, we reported two cases of recurrent oral herpes infection which can be misinterpreted as other diseases and were difficult to distinguish as primary or recurrent infections. The objective of this case report was to increase the dentist's knowledge in aetiology, clinical manifestations, diagnosis and treatment of the recurrent oral herpes infections in adult patient.

\section{CASE MANAGEMENT}

Patients were clinically evaluated before treatment and the measurement of the subjective intensity of pain was recorded using the visual analogue scale (VAS) at the time of examination.

\section{First Case}

A 41-year-old female patient came to the Integration Clinic, Universitas Indonesia (UI) Dental Hospital. She was hit by her child's head, which had caused several painful canker sores on her lower gingiva (VAS 7/10) for six days. She did not remember whether her sores were preceded by vesicles or not. She usually experiences canker sores for two to three times a year on the tongue, lips, palate and heal within one week without treatment. Before the sores appear, the patient claimed to have a fever for almost one week and had pharyngitis and pain when swallowing. The patient had a history of gastritis, hepatitis A and B, and had completed treatment for her hepatitis two years ago.

On extraoral examination, her left and right submandibular glands were palpable, soft and pain. Dry, desquamated and several reddish areas on her lower lip were found.
In intraoral examination, multiple ulcerations of the lower gingiva region 3.2, 3.3-3.4, 4.1-4.2 and 4.3 with diameter of $3 \mathrm{~mm}$ to $5 \mathrm{~mm}$, round, regular edges, clear borders, yellowish white base that were surrounded by erythema. The working diagnosis at the initial visit was a recurrent intra oral herpes and cheilitis. We prescribed topical petroleum jelly applied four times a day for her lips, chlorhexidine gluconate $0.2 \%$ swish four times a day@10 ml and multivitamin containing zinc once a day. The patient was then sent for complete peripheral blood examination and serological tests for hepatitis B and herpes viruses (IgG, IgM anti HSV-1 and HSV-2).

At the second visit (one week after the initial one), the patient still had not redeemed the prescribed medicine and laboratory test. On the intraoral examination, we found canker sores that was reducing in number and pain compared to the initial visit (VAS 4/10). She stated that the outer part of her vagina had vesicles and often feels itchy, red, especially in her periods. We instructed the patient to apply a gauze moistened by chlorhexidine gluconate $0.2 \%$ four times a day on her sores and continue to give her multivitamins containing zinc once a day. We gave topical petroleum jelly, to be applied on her lips four times a day.

Patient reported her complete peripheral blood examination by phone with result of haemoglobin $12.3 \mathrm{~g} / \mathrm{L}$, haematocrit $37.2 \%$, MCV 83.4fL, MCHC 33.1fL, platelets 238.000, IgG Anti HSV-1 negative (0.19), IgM Anti HSV-1 negative (0.35), IgG Anti HSV-2 negative (0.17), IgM Anti HSV-2 positive (1.30), HBsAg nonreactive and Anti $\mathrm{HCV} 0.070$ (nonreactive). All indicators were within normal limits except for IgM Anti HSV-2. Based on this, the working diagnosis should have been primary infection of HSV-2. However, based on history and clinical manifestations, we established the working diagnosis as recurrent intra oral herpes infection. 

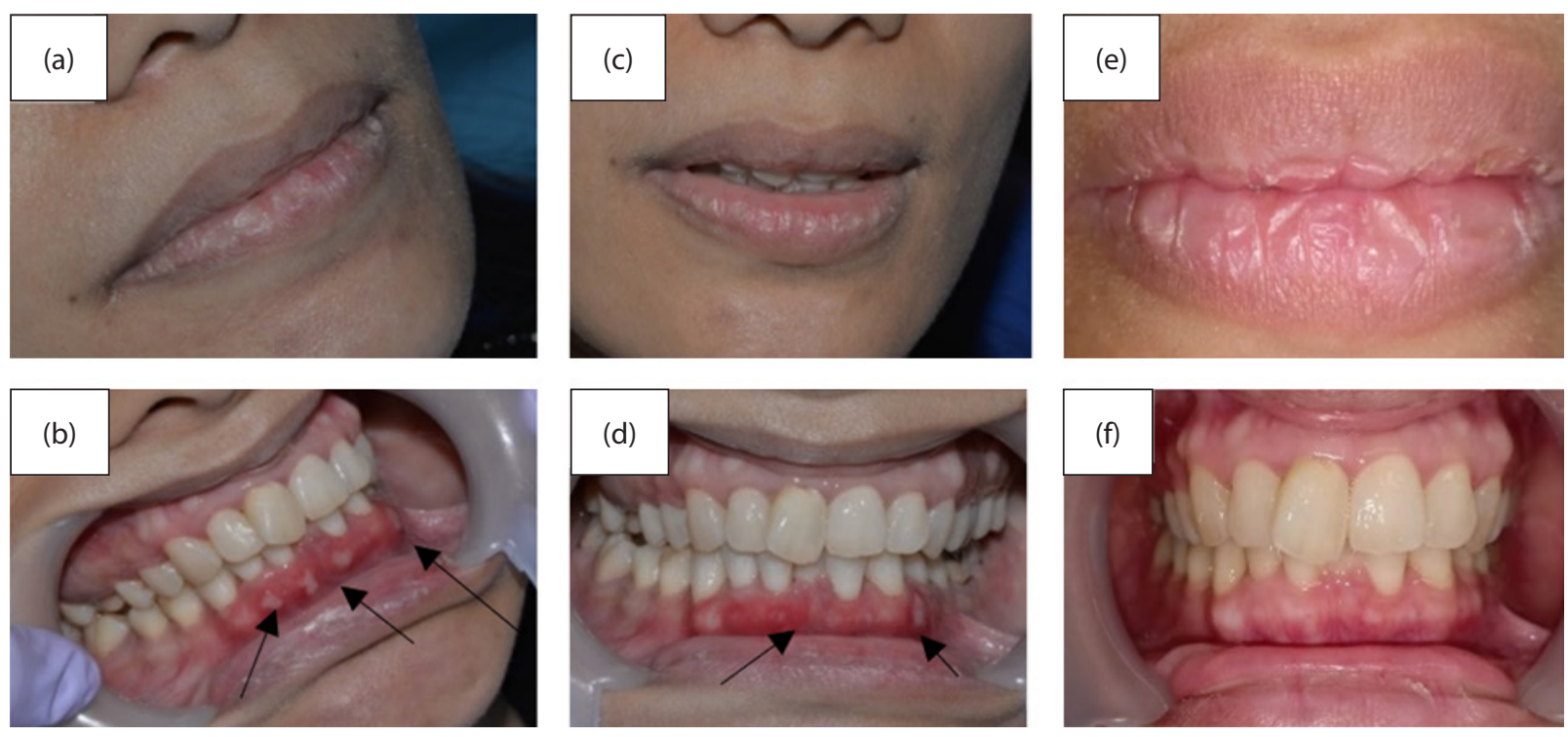

Fig. 1 First case as presented when the patient came on her: (a-b) first visit; (c-d) second visit; and (e-f) third visit.

On the third visit, the patient had used up all the prescribed medication. All medicines were used regularly according to the instructions given. Patient said that she rarely used the topical petroleum jelly on her lips. All the canker sores had healed. Patient was then given the information on intra oral herpes infection and its relationship with the laboratory test result, modes of transmission, symptoms in the oral cavity and possible of recurrence. Patient was also instructed to maintain her health condition, vulval hygiene and consult with the dermatoveneorologist if similar lesions appeared on her vagina. We managed her cheilitis with applied topical petroleum jelly four times a day.

\section{Second Case}

A 70-year-old male patient came to UI Dental Hospital with complaints of canker sores for five days. He felt pain in the entire mouth (VAS 7/10) and even felt severe headache. The patient was hospitalised for three days and received analgesic-antipyretic therapy, amlodipine, clindamycin, bisoprolol, injection of omeprazole, ranitidine and ceftriaxone. He was also instructed to apply gel containing triamcinolone acetonide on the canker sores area. After hospitalisation, he still felt pain in the mouth and his canker sores were not reduced.

According to the patient, these canker sores suddenly emerged in large numbers and was preceded by fever for three days. He denied any experience of canker sores before but claimed that his grandson had a history of canker sores. Analgesic, tantum verde and cefixime were used during the fever before the canker sores appeared. There was no rash, itching or vesicles in other parts of the body. The patient did not notice whether the canker sores were preceded by vesicles or not. There was no other disease as stated by the patient; however, he had high blood pressure during his hospitalised period. $\mathrm{He}$ claimed that he was very exhausted after traveling before the fever appeared.

On extraoral examination, we found a crust in the vermillion border of the upper lip (region 21) that was not painful. Intraorally, we found multiple ulceration of the labial mucosa on the lower lip, mandibular alveolar ridge, hard palate, right and left buccal mucosa, right and left lateral, dorsum and ventral tongue. All ulcerations were 

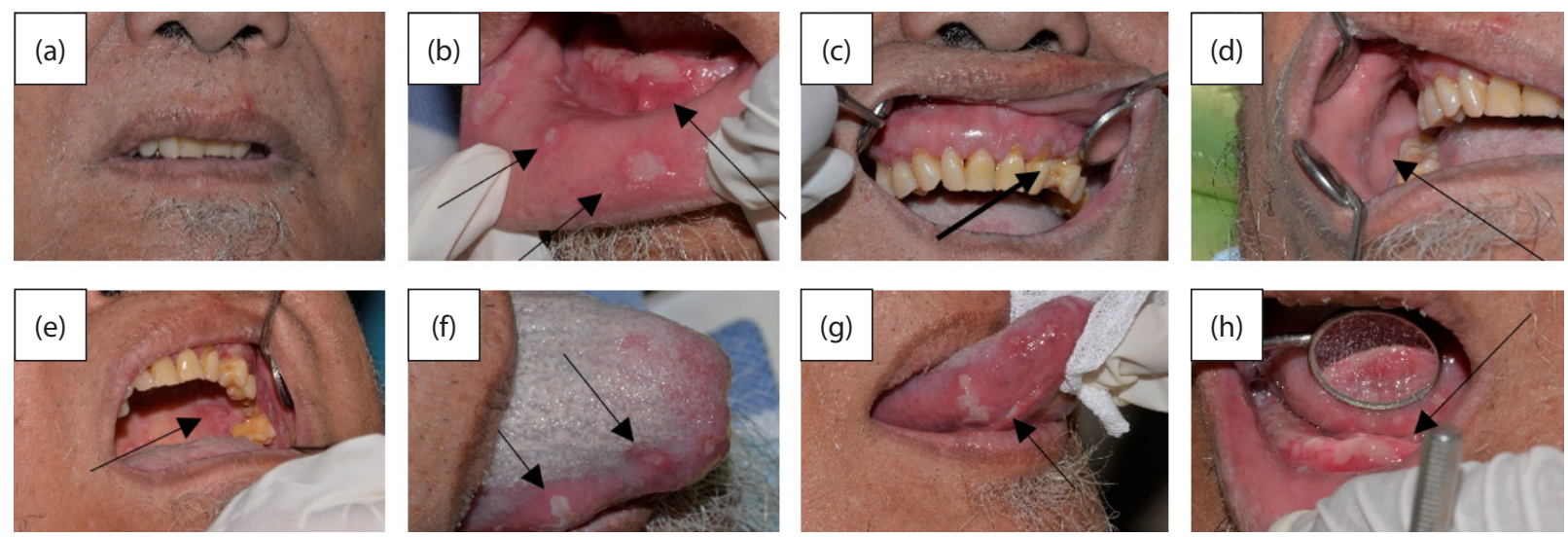

Fig. 2 Second case on the first visit: (a) Crust on upper vermillion borders of lips (region 21);

(b) Multiple ulcers on labial mucosa mandibular lips; (c) Desquamative areas on maxillary;

(d-e) Multiple ulcers on right and left buccal mucosa; $(f-g)$ Multiple ulcers on dorsal, lateral, and ventral of the tongue; and (h) Multiple ulcers extended to mandibular lingual gingival area.
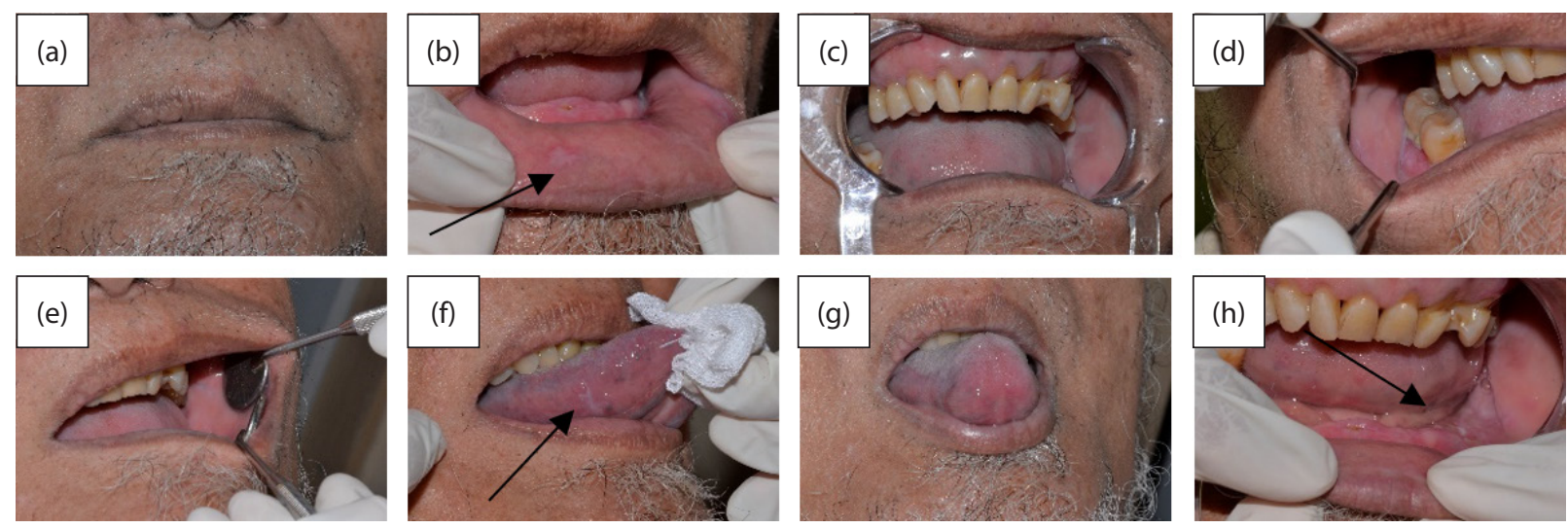

Fig. 3 Second case on the second visit. (a) Erythema on upper vermillion border of lips (region 21); (b) Multiple ulcers on healing in labial mandibular mucosa; (c) Healing on desquamative maxillary gingival area; $(\mathrm{d}-\mathrm{e})$ Healing ulceration on right and left buccal mucosa; $(\mathrm{f}-\mathrm{g})$ Ulceration on healing

in ventral and lateral of the tongue; and (h) Ulceration on healing in mandibular gingival area.

irregular, clearly defined edges, yellowish white base membrane with varying diameters between $1 \mathrm{~mm}$ to $8 \mathrm{~mm}$. We also found a desquamation area of the mandibular gingiva over the 1.3 to 2.4 region and the presence of erosive areas in the mandibular gingiva 4.3 to 4.6 region. The working diagnosis as based on the clinical examination was that a recurrent oral herpes infection. Patient was given information about the oral herpes infection, ways of transmission and how to prevent the recurrence. Patient was instructed to maintain good diet and rest. We also instructed the patient to gargle doxycycline mouthwash $100 \mathrm{mg}$ twice a day for $1 \mathrm{~min}$ to $2 \mathrm{~min} @ 10 \mathrm{ml}$ (for seven days) and was given supportive therapy with multivitamin containing zinc once a day.

Seven days after the initial visit, the patient felt improvement of his mouth. Pain score had been greatly reduced (VAS 2/10). The patient could eat and drink as usual. There were no more headaches reported. All the prescribed medicines were used up according to the instructions and multivitamins were taken daily. The patient stated that the canker sores had improved 

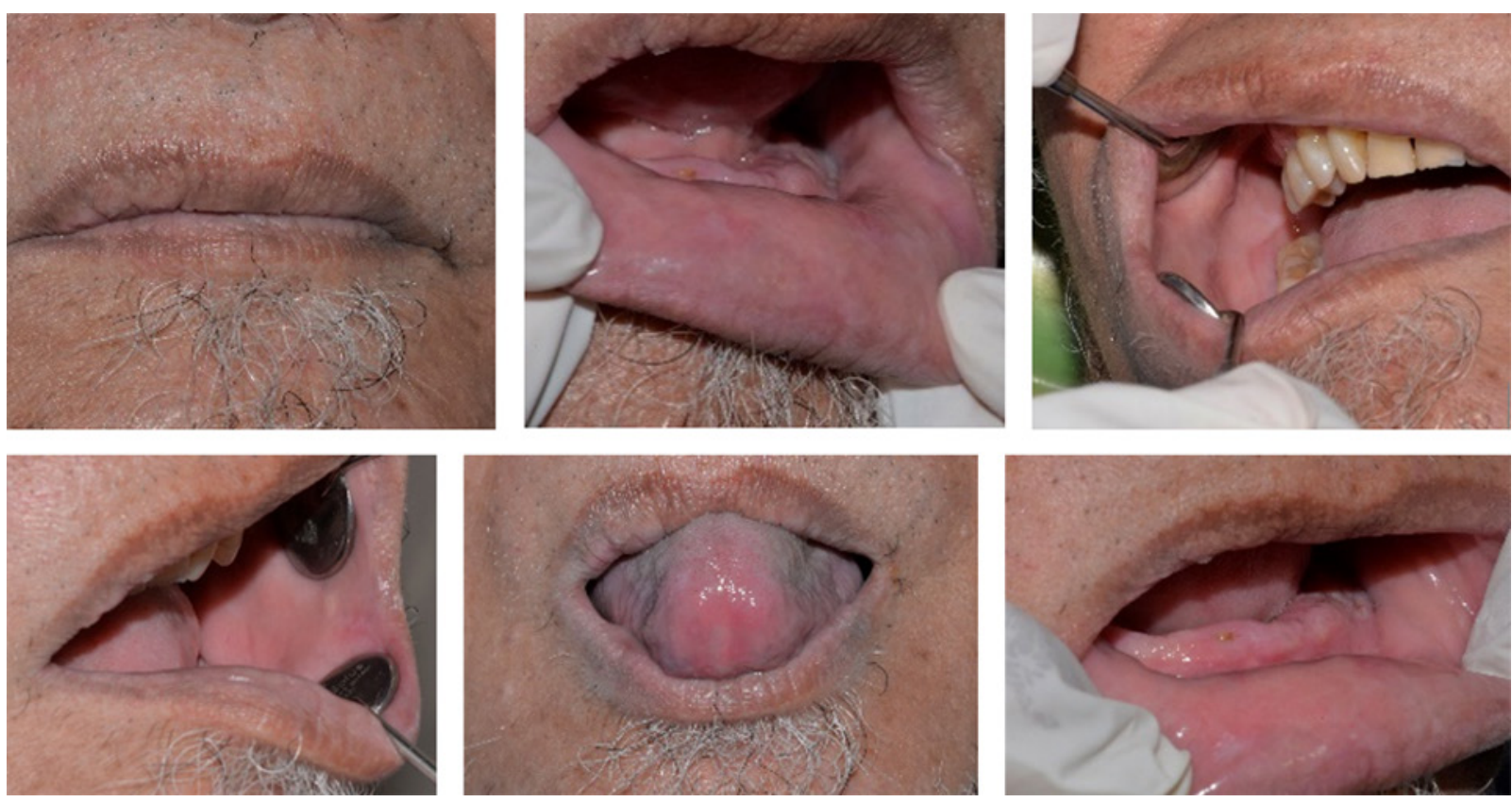

Fig. 4 Second case on the third visit. All the lesions had healed.

within three days of using the mouthwash. Because of the significant improvement, the doxycycline mouthwash therapy was reduced to once daily (at night). We also prescribed multivitamin containing zinc for seven days.

Fourteen days after the initial visit, the patient was completely cured, with no further complaints and was able to do activities as usual. Because of the recovery experienced by the patient, we stopped the doxycycline mouthwash and was replaced by gargle with warm salt water $(1 / 4$ tablespoon of salt dissolved in $250 \mathrm{ml}$ of boiled water) twice a day.

\section{DISCUSSION}

\section{First Case}

In the first case, herpes infection was considered as the working diagnosis because of the presence of prodromal symptoms prior to ulceration, located in the keratinised area and history of recurrent ulcers in the palate and gingiva. Viral infections can be distinguished from recurrent aphthous stomatitis based on its location. Viral infections are located in the keratinised area (vermilion border of the lip, hard palate, attached gingiva and alveolar ridge) while recurrent aphthous stomatitis is located in the non-keratinised area (Tilliss \& McDowell, 2002). In addition, viral infection is characterised by multiple ulcers with less delicate erythema area that surrounded the ulcers (Mortazavi et al., 2016). Primary herpes infection usually occurs in children and young adults with the presence of inflammation in the marginal and attached gingival areas so that the gingiva becomes erythematous, bleeding, and multiple small vesicles appear (Turton, 2017). Based on these, we chose the recurrent intra oral herpes infection as the working diagnosis. Possible triggering factor for this case was trauma (hit by her child's head). Local trauma to the oral cavity can result in recurrence of HSV (Siegel, 2002).

Although oral herpes infection diagnosis can made based on clinical manifestations, specific antibody tests are especially useful for diagnosing HSV-1 and HSV-2 infections in patient that showed symptomatic or asymptomatic symptoms (Morrow \& Friedrich, 2006). The patient in the present 
case was sent for complete peripheral blood examination and serological tests for hepatitis $\mathrm{B}$ and herpes viruses (HbSAg, IgG, IgM anti HSV-1 and HSV-2). Complete peripheral blood tests were carried out to determine whether there was involvement of nutritional factors in HSV virus reactivation (Panda \& Ding, 2015). The laboratory test results of the present case were within the normal limits except for Anti HSV-2 IgM (positive).

Medical practitioners mostly know HSV-2 as a virus that infects genital area, with little understanding or recognition of $\mathrm{HSV}-2$ manifestations in the oral cavity. HSV-2 can be a chronic infection and is usually more severe than HSV-1 (Burton, 2016; Putri \& Rahmayanti, 2018). There is a similar spreading type of HSV-2 and HSV-1 in the oral cavity even though HSV-2 is rarely isolated from lesions on the lips and oral cavity. During the primary infection phase, HSV-2 can cause lesions in extra genital areas that usually appear within two weeks after primary infection in fingers, buttocks, groin, or thigh. This extension into the genital area is caused by direct spread of the virus. In addition, aseptic meningitis can occur with symptoms such as fever, headache and photophobia (Schiffer \& Corey, 2009). In the oral cavity, HSV-2 can spread rapidly and manifest both as primary infection and secondary infection. In addition to sexual contact, HSV-2 can also be transmitted through other types of close contact such as sharing the eating utensils (Burton, 2016). Infections of the oral cavity associated with HSV-2 can also occur in the individuals with long-term immunosuppression, for example in cicatricial pemphigoid or HIV. The patient in the present case denied involving in any oral sex activities, so it can be concluded that HSV-2 transmission in the patient might develop from close contacts.

Interpretation of specific antibody tests for HSV infection are primary infections (positive $\operatorname{IgM}$ and/or positive viral culture accompanied by negative results on IgG at one time of examination), first nonprimary episodes (on IgG and $\operatorname{IgM}$ positive) and recurrence infection (positive IgG and negative $\operatorname{Ig} M$ ). Initially, the specific $\operatorname{Ig} M$ antibodies can only be detected after 10 days exposure to the virus and persisted for 7 to 10 days thereafter (Page et al., 2003). Sometimes in severe recurrence infections, $\operatorname{Ig} M$ antibodies can occur. Specific IgM antibodies have a low sensitivity and giving false positive results at low index values $(1.1-3.5)$. In the present case, anti-IgM value of HSV-2 is 1.30 which can provide false positive results; and specific $\operatorname{IgM}$ antibody can occur in recurrent infection (Biškup et al., 2015; Johnston \& Corey, 2016). Therefore, laboratory results alone cannot be used as single consideration of diagnosis. Based on the clinical manifestation, we recognised recurrent intra-oral herpes as the working diagnosis.

We treated the patient in the first case by applying gauze moistened by chlorhexidine gluconate $0.2 \%$ four times a day and by giving multivitamin containing zinc once a day. Patient was also treated with topical petroleum jelly four times a day for her cheilitis. In the second visit, one week after the patient's initial visit, there was a decrease in number and size of the ulcer even though the patient did not use the prescription medicine. This happened because the herpes infection is a self-limiting disease that can heal by itself in immunocompetent individual (Ballyram et al., 2016). Conventional therapy for oral lesions associated with HSV infection is acyclovir (either in oral form or topical), but due to poor gastrointestinal absorption and bioavailability, this therapy is less commonly used (Chauvin \& Ajar, 2002). Considering the severity of disease and immunocompetent conditions in the patient, we managed her with chlorhexidine gluconate $0.2 \%$ and multivitamins containing zinc. This also supports the theory which states that therapy with acyclovir should be given in the initial phase (less than five days from prodromal symptoms appear). Thus, the recommended therapy is the nonspecific therapy and supportive therapy using 
mouthwash containing antimicrobials such as chlorhexidine. Multivitamins containing zinc is given because zinc can inhibit replication of HSV-1 and HSV-2 (Shah et al., 2014).

\section{Second case}

In the second case, there was a complete ulceration in the keratinised and nonkeratinised mucosa with erosive area in the mandibular gingiva accompanied by prodromal symptoms. The overall mandibular gingiva appeared to be erythematous. The patient appeared to be extremely sick and even had severe headaches. These clinical manifestations resembled the primary infection of HSV specifically, the presence of severe symptoms, fever, malaise, marginal gingivitis (sometimes accompanied by gingival hyperplasia), inflammation of the margin and attached gingival area and widespread vesicular eruption which became the ulcerated area of the labial mucosa, tongue, buccal and vestibular mucosa, hard palate, soft palate, floor of the mouth, tonsillar mucosa and pharyngeal (Turton, 2017).

In this patient we found a crust in vermillion border of upper lip (region 21). This crust is a characteristic symptom of recurrent herpes labialis infection. Recurrent herpes labialis infection is characterised as a small, erythematous, and multiple papules which then develops into vesicle clusters that will rupture within two days and form crusts within three to four days. HSV recurrent infections can appear as a recurrent form of herpes labialis or recurrent intra oral herpes infection. This recurrent infection represents the reactivation process of a latent virus in the nerve but not HSV virus reinfection (Siegel, 2002).

In immunocompromised individuals, recurrent herpes infection can appear throughout the oral mucosal surface including in the non-keratinised area. This infection can also appear as a single lesion on the dorsal surface of the tongue
(Basavarajappa et al., 2015). Our patient is 70 years old, which is classified as an elderly individual with declining body defence mechanism. Based on the above considerations, the working diagnosis in this case was recurrent herpes infection in the oral cavity.

In the present case, we found massive erosive and ulceration in the edentulous area of the mandibula. Recurrence of the HSV can occur in traumatic area of mastication (Siegel, 2002). The patient also stated that a few days before the prodromal symptoms he felt very exhausted due to traveling. This condition can be one of the triggering factors in HSV recurrence. Severe ulceration experienced by the patients can also be aggravated by topical use of steroids (triamcinolone acetonide). Topical steroids applied to herpes lesions in the oral cavity should be avoided because it will cause rapid spreading of the virus. Failure to recognise viral infections in the early stages might cause patients to get unnecessary high dose of antibiotics.

Our management in the second case was the administration of $100 \mathrm{mg}$ of doxycycline mouthwash twice a day @ $10 \mathrm{ml}$. Doxycycline dose than was tapered down once a day due to the improvement of the patient's condition. The use of doxycycline in ulcerated lesions can inhibit MMP (Metalloproteinase matrix). In the subepithelial layer, MMP can promote disruption in the connection between the epithelium and connective tissue that cause sloughing of the epithelium and ulcer formation (Preshaw et al, 2007).

\section{CONCLUSION}

Herpes infection in the oral cavity can mimic other types of ulceration that occur in the oral cavity. The presence of prodromal symptoms, the shape and location of ulcer are particularly useful in determining diagnosis. There are two types 
of herpes infections that can occur in the oral cavity. They are primary and recurrent form infections. In few cases there can be similarities in the clinical manifestations between the two types of infection. Assessment of the patient's immunity status and severity of infection will ensure better management of the HSV virus infection.

\section{REFERENCES}

Ballyram R, Wood NH, Khammissa RAG, Lemmer J, Feller L (2016). Oral diseases associated with human herpes viruses: Aetiology, clinical features, diagnosis and management. South Afr Dent F, 71(6): 253259.

Basavarajappa S, Ramakrishnaiah R, Durgesh B, Al Kheraif AA, Devang Divakar D (2015). Oral infections of herpes simplex virus: Symptoms, diagnosis, treatment and pathophysiology in periodontal disease. Rev Med Microbiol, 26(4): 138-142. https://doi .org/10.1097/MRM.0000000000000042

Bhateja S, Arora G, Mastud SK (2017). Recurrent intraoral herpes (RIH) infection - A case report. Biomed f Sci Tech Res, 1(3): 549-551. https://doi.org/10.26717/ BJSTR.2017.01.000241

Biškup UG, Uršič T, Petrovec M (2015). Laboratory diagnosis and epidemiology of herpes simplex 1 and 2 genital infections. Acta Dermatovenerol Alp Pannonica Adriat, 24(2): 31-35. https://doi.org/10.15570/ actaapa. 2015.9

Burton ZF (2016). Herpes simplex virus serotype 2 oral infection. FSM Biochem Mol Biol, 3(1): 1015 .

Chauvin PJ, Ajar AH (2002). Acute herpetic gingivostomatitis in adults: A review of 13 cases, including diagnosis and management. f Can Dent Assoc, 68(4): 247-251.
Johnston C, Corey L (2016). Current concepts for genital herpes simplex virus infection: Diagnostics and pathogenesis of genital tract shedding. Clin Microbiol Rev, 29(1): 149-161. https://doi.org/10.1128/CMR $.00043-15$

Morrow R, Friedrich D (2006). Performance of a novel test for IgM and IgG antibodies in subjects with culture-documented genital herpes simplex virus-1 or -2 infection. Clin Microbiol Infect, 12(5): 463-469. https://doi.org/10.1111/j.1469-0691.2006 $.01370 . x$

Mortazavi H, Safi Y, Baharvand M, Rahmani S (2016). Diagnostic features of common oral ulcerative lesions: An updated decision tree. Int $\mathcal{F}$ Dent, 2016: 7278925. https://doi.org/10.1155/2016/7278925

Page J, Taylor J, Tideman RL, Seifert C, Marks C, Cunningham A et al. (2003). Is HSV serology useful for the management of first episode genital herpes? Sex Transm Infect, 79(4): 276-279. https://doi.org/10.1136/ sti. 79.4 .276

Panda S, Ding JL (2015). Natural antibodies bridge innate and adaptive immunity. f Immunol, 194(1): 13-20. https://doi.org/ 10.4049/jimmunol.1400844

Preshaw PM, Grainger P, Bradshaw MH, Mohammad AR, Powala CV, Nolan A (2007). Subantimicrobial dose doxycycline in the treatment of recurrent oral aphthous ulceration: A pilot study. F Oral Pathol Med, 36(4): 236-240. https://doi.org/10.1111/j .1600-0714.2007.00507.x

Putri AAM, Rahmayanti F (2018). Management of acute (primary) herpetic gingivostomatitis in immunocompetent adult patient: A case report. Proceedings of the International Dental Conference of Sumatera Utara 2017 (IDCSU 2017). Dordrecht: Atlantis Press, pp. 30-33. https://doi.org/10.2991/idcsu-17.2018.9

Schiffer JT, Corey L (2009). New concepts in understanding genital herpes. Curr Infect Dis Rep, 11(6): 457-464. https://doi.org/ 10.1007/s1 1908-009-0066-7 
Shah S, Devi MP, Ravindra SV, Tyagi K, Singh D (2014). Primary herpetic gingivostomatitis: A case report and review of literature. TMU J Dent, 1(3): 119-124.

Siegel MA (2002). Diagnosis and management of recurrent herpes simplex infections. f Am Dent Assoc, 133(9): 1245-1249. https://doi.org/10.14219/jada.archive. 2002 .0366

Tilliss TS, McDowell JD (2002). Differential diagnosis: Is it herpes or aphthous? f Contemp Dent Pract, 3(1): 1-15.
Turton M (2017). A case report on symptomatic primary herpetic gingivostomatitis. F Dent Health Oral Disord Ther, 8(8): 00317. https://doi.org/10.15406/jdhodt.2017.08 .00317

van der Plas H, Hardie D (2010). Herpes simplex virus 1 and 2: A therapeutic approach. South Afr $\mathcal{J}$ Epidemiol Infect, 25(3): 5-9. https://doi.org/10.1080/10158782.2010 .11441392 